\title{
Sistema de control para un sistema solar acondicionador de aire por absorción utilizando simulación en TRNSYS
}

Miguel Chen Austin ${ }^{1-3}$ (D), Rhona Díaz 1 (iD), Ignacio Chang ${ }^{1,3}$ (iD), Julio Rodríguez ${ }^{1}$

1 Universidad Tecnológica de Panamá, 2 Grupo de Investigación en Energética y Confort en Edificaciones Bioclimáticas (ECEB), Universidad Tecnológica de Panamá, Facultad de Ingeniería Mecánica; ${ }^{3}$ Grupo de Investigación Sistemas de Control Inteligente e Informática Industrial (SCIII), Universidad Tecnológica de Panamá, Facultad de Ingeniería Eléctrica

miguel.chen@utp.ac.pa, rhona.diaz@utp.ac.pa, ignacio.chang@utp.ac.pa, julio.rodriguez@utp.ac.pa

DOI: 10.33412/pri.v12.1.2378

\section{이이(2)}

Resumen: Este trabajo de investigación, tiene como objetivo mejorar el desempeño de una instalación solar de acondicionamiento de aire por absorción, por medio del diseño de un sistema de control simple, para fácil implementación. Esta instalación fue diseñada por E. Quintana (2012) en TRNSYS, un programa de simulación de sistemas. Con la visión de una futura implementación, un análisis de desempeño del Chiller de absorción se llevó a cabo en base a los resultados de la simulación del trabajo precedente. Se encontró que su desempeño no alcanza los rangos de operación segura dentro del tiempo de operación diaria, cada vez que el sistema se inicia. Basado en esto, el trabajo propuesto abarca el diseño de un sistema de control que permita mejorar el desempeño del Chiller de absorción, desde el punto de vista de seguridad de éste último y la reducción del consumo eléctrico de la instalación. La operación segura del Chiller se alcanza cuando éste y todo el sistema, operan dentro de los rangos nominales indicados por el fabricante. Los resultados indicaron que los parámetros a optimizar son las temperaturas del agua que entra al generador y al acumulador térmico. Empleando las estrategias de control diferencial On-Off y Feedback, los resultados de las simulaciones muestran que el primer objetivo fue alcanzado. Por el contrario, el segundo, no se logra alcanzar ya que la energía proveniente de la fuente solar no logra suplir las necesidades del Chiller dentro del tiempo de operación diaria.

Palabras claves: chiller de absorción, control diferencial, control Feedback, instalación solar, optimización, seguridad operacional, Simulación en TRNSYS.

Title: Control System of a Solar System Air Conditioner by Absorption using Simulation in TRNSYS
Abstract: This research aims to improve the performance of a solar-absorption-air-conditioning installation, through the design of a simple control system, for easy implementation. This installation was designed by E. Quintana (2012) in TRNSYS, a systems simulation program. With the vision of a future implementation, a performance analysis of the absorption Chiller was carried out based on the results of the simulation from the previous work. It was found that its performance does not reach safe operating ranges within the daily operation time, every time the system starts. Based on this, the proposed research includes the design of a control system to improve the performance of the absorption chiller, from the safety point of view of the latter and the reduction of the electrical consumption of the installation. The safe operation of the Chiller is achieved when it and the whole system, operate within the nominal ranges indicated by the manufacturer. The results indicated that the parameters to be optimized are the temperatures of the water entering the generator and the thermal accumulator. Using the On-Off and Feedback differential control strategies, the results of the simulations show that the first objective was reached. In contrast, the second, could not be achieved, since the energy from the solar source fails to meet the needs of the Chiller within the daily operating time.

Key words: Absorption Chiller, Solar Installation, Differential Control, Feedback Control, Optimization, operational safety, Simulation in TRNSYS.

Tipo de artículo: Investigación.

Fecha de recepción: 3 de mayo de 2020.

Fecha de aceptación: 5 de enero de 2021.

\section{Introducción}

El proceso de refrigeración y/o acondicionamiento de aire es uno de los procesos que más consume electricidad en la República de Panamá [1],[2]. Debido a esto, se está en la búsqueda continua de nuevas alternativas que ayuden a disminuir este consumo. Actualmente, se están desarrollando estudios sobre sistemas de absorción y su aplicación con colectores solares térmicos, bajo las condiciones ambientales de Panamá. Sin embargo, estos sistemas necesitan de un sistema de control adecuado, para funcionar en óptimas condiciones.

La primera propuesta de estudio e implementación fue realizada y desarrollada por la Ing. Rhona Díaz, del Centro de Investigación e Innovación Eléctrica, Mecánica y de la Industria (CINEMI) de la Universidad Tecnológica de Panamá (Sede Tocumen). Este estudio, pretende evaluar la opción de la utilización de un nuevo sistema para Panamá en el ámbito del acondicionamiento de espacios, con el proyecto titulado: "Evaluación del Uso de Colectores Solares Térmicos en Sistemas de Absorción para Producción de Aire Acondicionado y/o Refrigeración en Panamá", desarrollado con fondos de la Secretaría Nacional de Ciencia, Tecnología e Innovación (SENACYT). 
Primeramente, se diseñó el sistema solar por absorción que se utilizó en el proyecto. Este diseño fue desarrollado por E. Quintana en 2012 [3], utilizando la herramienta de simulación TRNSYS (Transient System Simulation).

El presente trabajo tiene como objetivo general, diseñar un sistema de control para dicho sistema solar acondicionador de aire por absorción y optimizar su rendimiento, utilizando la misma herramienta de simulación.

A la fecha, en Panamá no se conocen empresas que diseñen y/o fabriquen equipos como Chiller de absorción (o Chillers de absorción). Los equipos de este tipo son, por lo general, importados desde USA o Alemania. Por lo tanto, estos equipos ya están diseñados bajo las condiciones ambientales del lugar donde se fabricó; que muchas veces no concuerdan con las condiciones ambientales de Panamá. Por este motivo, se hace necesario diseñar un sistema de control que ayude a modificar variables en el sistema, que permita la opción de encontrar un rango de valores para los cuales el sistema trabaje de forma óptima.

\subsection{Controles en instalaciones solares}

En la gran mayoría de los sistemas de control en las industrias de procesos (aproximadamente el 90\%), todavía se utilizan controladores PID. El cual ha sido accesible, comercialmente, por más de 60 años. A continuación, se describen los más utilizados [4]:

a. Estrategias de control convencionales: control ProporcionalIntegral-Derivativo (PID), control Feedforward (FF) y el control cascada (CC),

b. Técnicas clásicas del control avanzado: Gain scheduling (GS) y tiempo de compensación de retardo (TDC),

c. Técnicas extensamente utilizadas del control avanzado: control predictivo (MPC), control interno (IMC) y el control adaptivo (AC),

d. Nuevas técnicas del control avanzado: control óptimo (LQG), control no lineal (NC), control robusto (RC), controladores de red neural (NNC) y el control en lógica difusa (FLC).

\subsection{Controles en instalaciones acondicionadoras de aire solar}

Para el año 2011, Núñez-Reyes y Bordons [5] encontraron que una de las necesidades principales del control en estos sistemas, es suministrar agua enfriada (a la temperatura requerida) a los sistemas de distribución de aire para lograr acondicionar el recinto o habitación.

Esto se logra controlando la temperatura del agua caliente suministrada por el campo de colectores solares. Ya que la energía primaria no es manipulable, la temperatura deseada se consigue actuando sobre el caudal circulante. La contribución solar, además de la radiación estacional y las variaciones cíclicas diarias, depende de las condiciones atmosféricas como la cobertura de las nubes, la humedad, así como la transparencia del aire. Es importante mantener una temperatura de salida constante, ya que las condiciones solares cambian. Y el único medio disponible para lograr esto es mediante el ajuste del caudal.

Para lograr el objetivo mencionado anteriormente, el sistema de control debe mantener la temperatura de salida del fluido portador del calor al nivel deseado a pesar de las perturbaciones como los cambios en el nivel de radiación solar (causado por las nubes), la reflectancia del vidrio o la temperatura de entrada del agua. Esto es logrado, manipulando una válvula de tres vías, que varía el caudal a través del campo de colectores. El mantenimiento de una temperatura de salida constante a lo largo del día, debido a que las condiciones solares cambian, requiere una amplia variación en el nivel de flujo operativo.

Por otro lado, uno de los objetivos propuesto por Bujedo et al. 2011 [6], lo fue comparar diferentes estrategias de control aplicadas a un sistema real, con tres años de estudio de experimentales (verano de 2005 hasta 2007). Las tres estrategias de control comparadas fueron: (a) Control Convencional: El refrigerador trabaja a plena carga en todo momento, por lo que la regulación se basa en la conmutación de la enfriadora y sus bombas de alimentación de encendido y apagado. (b) Adaptación de las temperaturas del condensador: En este caso, las temperaturas del condensador son adaptadas a las del generador y la carga será regulada por ciclos de encendido y apagado, y (c) Control dual en el condensador-generador: Como en el segundo caso, en la regulación de la temperatura de condensación basada en el generador y la carga de enfriamiento será ajustada por el control del flujo en el generador. En la descripción del desarrollo de las estrategias, se determinó que el mejor resultado para la instalación fue obtenido cuando se trabaja en forma dual con el generador y el condensador.

Se menciona, además, que se pueden obtener mejores resultados, si algunos defectos del sistema que han sido detectados se resuelven. Defectos tales como: (a) la producción y la demanda deben coincidir en el tiempo, (b) la capacidad y demanda del refrigerador deben estar correlacionadas, (c) la regulación del flujo másico del generador debe realizarse usando bombas de flujo variable en vez de utilizar válvulas de tres vías, las cuales perturban la estratificación de los tanques de almacenamiento solar, y (d) la conexión hidráulica entre los colectores de un determinado campo y el encuentro de ambos campos, no debe ser balanceado por válvulas si el sistema es operado dinámicamente con bombas de velocidad variable. Esto creará diferencias en los flujos que pasan a través de cada ramal de los colectores, reduciendo la eficiencia colectiva e individual de los colectores.

Resultados de otra investigación, Zhai et al. 2011 [7], muestran que durante los días nublados el sistema no podría proveer un efecto de enfriamiento, cuando opera convencionalmente. Sin embargo, en condiciones de carga parcial, el refrigerador (Chiller) podría ser energizado, usando energía solar como fuente de calor. Los componentes principales de una máquina de absorción solar son el campo de colectores solares, el refrigerador por absorción (Absorption Chiller) y el tanque de almacenamiento de agua caliente. El rendimiento completo del sistema depende de la unión de estos tres componentes. Generalmente, un COP de 0,6 puede ser obtenido 
bajo las condiciones de diseño. También, de acuerdo con los resultados, los sistemas de refrigeración solar siempre incluyen el tanque de almacenamiento de agua caliente, el cual acumula calor por la circulación de captación solar. En conjunto, el volumen específico del tanque fue establecido entre 0,01 y $0,08 \mathrm{~m}^{3}$ por cada metro cuadrado de colectores. Para los sistemas de refrigeración solar que sólo operan en modo de refrigeración, como los del área tropical, se recomienda utilizar los valores más bajos de volumen específico. Finalmente, se indica que los sistemas de refrigeración solar no podrían competir con los sistemas estándar de refrigeración. La tecnología de refrigeración solar no es económicamente factible sin subsidio, principalmente por su alto costo de inversión. Los sistemas de refrigeración solar pueden ser utilizados como un sistema autónomo o acompañados de un sistema convencional de aire acondicionado, para mejorar la calidad del aire interior. A manera de incrementar la tasa de utilización y la fracción solar, todos los sistemas de refrigeración solar son multifuncionales y son usados para suplir calor y agua caliente en otras temporadas en países templados. Mientras que, generalmente, en países tropicales estos sistemas tienen un uso durante todo el año. La disponibilidad del recurso solar es otro factor importante en la determinación de la tecnología de refrigeración solar más adecuada para cierta localización. Se recomienda que los sistemas auxiliares de energía de los sistemas de refrigeración solar sean otra energía renovable o bombas de calor de alta eficiencia. Además, es altamente recomendable establecer una estrategia operacional óptima de control, para realizar un suave cambio entre el sistema de refrigeración solar y el sistema auxiliar de energía.

\section{Metodología}

A continuación, se presenta la metodología empleada. Primeramente, se describe el sistema de acondicionamiento de aire solar por absorción estudiado, seguido por un análisis general de los parámetros optimizables, definiendo las funciones objetivo dentro de dicho sistema, a través de un sistema de control. Luego se presentan las estrategias de control escogidas, dentro de las limitaciones del programa TRNSYS, y sus diseños para ser aplicadas al sistema de acondicionamiento de aire.

\subsection{Descripción del sistema de acondicionamiento de aire solar por absorción al cual se le diseñará el sistema de control}

El sistema acondicionador de aire solar por absorción que se simuló, Quintana 2012 (ver esquemático en la figura 1 y 2), consta de cuatro circuitos hidráulicos. El primero: colectores - Tanque de almacenamiento, lo componen el campo de colectores; con un área de captación solar de 150,66 $\mathrm{m}^{2}$, una bomba de agua caliente y la válvula de alivio, cerrando con el tanque de almacenamiento. El segundo: tanque de almacenamiento Absorbedor, lo componen el tanque de almacenamiento, el cual posee una fuente auxiliar de calor interna, una bomba de agua caliente, una válvula de tres vías, una tubería tee, cerrando con el absorbedor. Los dos anteriores conforman el circuito de agua caliente. El tercero: Absorbedor - Torre de enfriamiento, lo componen la torre de enfriamiento y una bomba de agua fría. Por último el cuarto circuito: Absorbedor - Fan Coils, lo ocupan una serie de tuberías tee (diverter), la bomba de agua fría y cuatro unidades fan coil, uno para cada zona a acondicionar [3].

Así pues, nos enfocaremos primeramente en el establecimiento de los parámetros que pueden ser optimizados en estas instalaciones.

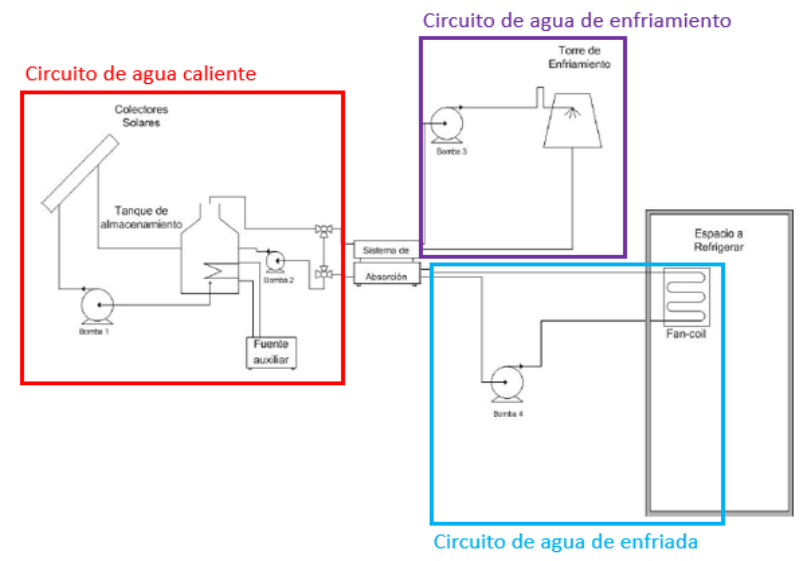

Figura 1. Esquema general de la instalación [3].

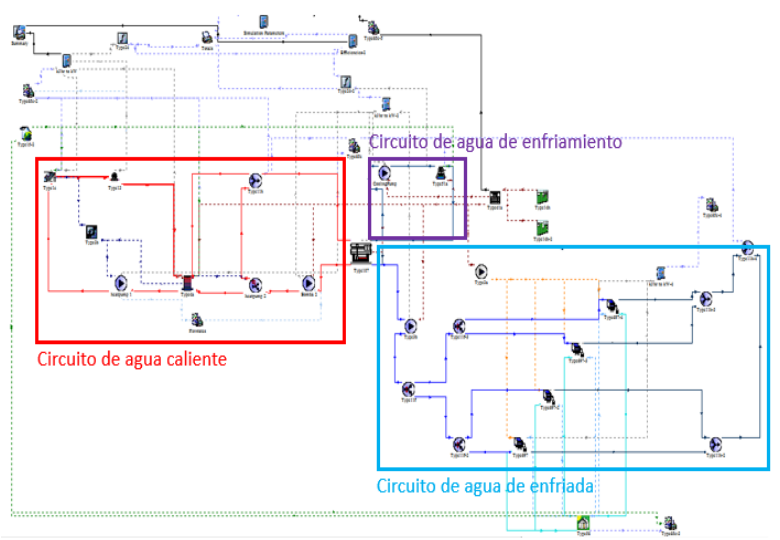

Figura 2. Instalación solar en TRNSYS [3].

\subsection{Análisis de parámetros optimizables dentro de las instalaciones de aire acondicionado solar por absorción}

Para corroborar que el sistema es en efecto optimizable, recurrimos al uso del análisis de grados de libertad: Un sistema con $\mathrm{M}$ ecuaciones, $\mathrm{N}$ variables y $\mathrm{F}$ grados de libertad, será optimizable si y sólo si $F>1$ dada la expresión (1).

$$
F=N-M
$$

Esta expresión debe ser utilizada luego de haber escrito todas las ecuaciones correspondientes al modelo matemático de la fisicoquímica del proceso. En nuestro caso, se tienen las siguientes relaciones:

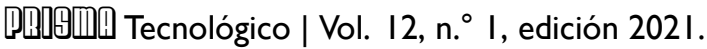


- Balances de masa,

- Balances de energía,

- Balances de momentum o fuerzas,

- Relaciones termodinámicas,

- Restricciones de diseño.

La determinación de las ecuaciones correspondientes a los puntos anteriores, para nuestro proceso en particular (ver figura 1) y para cada dispositivo, dieron como resultado un total de 52 variables y 44 ecuaciones. Lo cual confirma que el sistema es en efecto, optimizable.

Ahora, se prosigue a determinar todos los parámetros que pueden ser optimizados en estos procesos. Luego de una detallada revisión literaria, los siguientes parámetros son los más propensos a ser optimizados:

- El flujo másico en los colectores para maximizar la extracción de exergía,

- La temperatura del agua caliente que entra al generador,

- El ángulo de inclinación de los colectores solares,

- El tamaño del tanque de almacenamiento de calor,

- El consumo eléctrico de la instalación (calentador auxiliar, bombas, ventiladores, etc.),

- Costos asociados al consumo eléctrico,

- La estabilidad y el tiempo de respuesta de las temperaturas,

- El consumo de agua,

- Eficiencia y COP del sistema.

Teniendo lo anterior en cuenta, luego de un estudio de criticidad, los parámetros escogidos para optimización son los siguientes:

- La estabilidad y tiempo de respuesta de las temperaturas del agua caliente, de enfriamiento y enfriada, para garantizar operación segura del Chiller de absorción.

- Disminuir el consumo eléctrico, que se obtiene en los resultados de [3].

La primera de la lista es extremadamente necesaria; ya que, si el Chiller de absorción no opera dentro de los rangos nominales, se pueden encontrar problemas de cristalización del $\mathrm{LiBr}$ dentro de sus tuberías, obstruyendo el paso de la mezcla $\mathrm{LiBr}-\mathrm{H} 2 \mathrm{O}$.

Observando los resultados (ver figura 7), se muestra el comportamiento de las tres temperaturas más importantes: $T_{G}$ temperatura del agua que entra al generador, $T_{E}$ temperatura del agua enfriada, $T_{T}$ temperatura del agua de enfriamiento; podemos notar que las únicas dos temperaturas que presentan picos fuera del comportamiento normal (valores nominales), es $T_{E}$ que sale del Chiller y $T_{G}$. Esta última, se encuentra en un rango de temperaturas no acorde con las especificaciones del fabricante $\left(83^{\circ} \mathrm{C} \leq T_{G} \leq 95^{\circ} \mathrm{C}\right)$. Nótese que en los momentos donde aparecen los picos en $T_{E}, T_{G}$ tiene un valor por debajo de $80^{\circ} \mathrm{C}$, esto indica que al generador no le llega el agua a la temperatura adecuada en esos momentos (ej.: al comienzo del día) y esto a su vez, ocasiona la aparición de los picos en $T_{E}$.

Por otra parte, la presencia de estos picos indica también que, en esos momentos del año, la carga térmica del recinto $\left(\dot{Q}_{R}\right)$ que se quiere acondicionar, sobrepasa la capacidad nominal de enfriamiento $\left(\dot{Q}_{C}\right)$ del Chiller de absorción.

Con la finalidad de optimizar el menor número de variables posibles, se hace necesario conocer la relación entre las mencionadas en el párrafo precedente: $T_{E}, T_{G}, \dot{Q}_{R}$ y $\dot{Q}_{C}$, lo que reducirá el diseño del sistema de control. La relación entre 3 de las variables mencionadas, es la siguiente [3]:

$$
\dot{Q}_{C}=0,42 *\left(T_{G}-2,5 * T_{T}+1,8 * T_{E}\right)+0,9
$$

Donde $T_{T}$ representa el agua de enfriamiento que proviene de las torres de enfriamiento.

La ecuación (2) se determina a partir de la conocida relación: ecuación característica del Chiller, la cual es única para cada Chiller de absorción y debe ser incluida en la data del fabricante del equipo. Por último, $\dot{Q}_{C}$ y $\dot{Q}_{R}$ se relacionan por medio de un balance de energía en el serpentín de la unidad FanCoil que se encuentra al interior del recinto a acondicionar. En un caso ideal, se tiene:

$$
\dot{Q}_{R}=\dot{Q}_{C}
$$

Así se puede concluir que, si $T_{G}$ se mantiene dentro del rango nominal, $T_{E}$ se mantendrá también dentro de este rango y, por lo tanto, $\dot{Q}_{C}$ y $\dot{Q}_{R}$ también. Claro está, que $T_{T}$ se debe también tener en el rango nominal que solicita el Chiller de absorción. De esta manera, si $T_{T}$ se mantiene dentro del rango nominal, $\dot{Q}_{C}$ y $T_{E}$ dependerán solo de $T_{G}$. Aquí, se asume que $T_{T}$ se logra mantener dentro del rango nominal, por medio de las torres de enfriamiento.

Dado lo anterior, se obtienen como funciones objetivo y parámetros a optimizar: minimizar el tiempo de respuesta en que $T_{G}$ llega a su valor nominal,

$$
\min \left(t_{T_{G} \rightarrow T_{G, \text { nominal }}}\right) \rightarrow 0
$$

y, reducir la amplitud de $T_{G}$, manteniéndola dentro de los valores nominales:

$$
\min \left(A_{T_{G}}\right) \rightarrow A_{T_{G, \text { nominal }}}
$$

Desde el punto de vista de la seguridad del Chiller, se hace críticamente necesario que el agua que llega al generador se encuentre dentro de sus valores nominales, ya que se pueden ver afectadas las reacciones químicas que se dan dentro del Chiller de absorción. La temperatura que llega al generador proviene del circuito secundario de agua caliente; es entonces que, es aquí en donde se propuso colocar un controlador diseñado de tal manera que permita cumplir lo expresado por las expresiones (4) y (5). 
En lo que respecta al otro parámetro que se decidió optimizar, primeramente: el consumo eléctrico del calentador auxiliar del tanque de almacenamiento (o acumulador) del circuito de agua caliente; lo anterior resta imposible de realizar, en momentos donde la energía proveniente de los colectores solares no es la suficiente para garantizar la temperatura necesaria del agua que entra al generador. Sin embargo, decidimos también, diseñar un controlador que permita optimizar el uso de este calentador auxiliar. Este último se logra reducir, cuando la temperatura superior del acumulador de calor se mantiene cercana o igual a la temperatura deseada a la entrada del generador a un caudal específico. Lo anterior se deduce de la ecuación para la potencia térmica del calentador:

$$
\dot{Q}_{\text {aux }}=c_{P} * \rho * \dot{V} *\left(T_{G, \text { nominal }}-T_{\text {acumulador }}\right)
$$

Donde $c_{P}$ y $\rho$ son propiedades del agua, y $\dot{V}$ representa el caudal o flujo volumétrico del acumulador.

De esta manera nos queda que $\min \left(\dot{Q}_{\text {aux }}\right) \rightarrow 0$, se hace posible cuando $T_{G, \text { nominal }} \approx T_{\text {acumulador }}$ a un $\dot{V}$ dado. Esto último indica entonces que es de suma importancia prestar atención a la $T_{\text {acumulador }}$. Del circuito primario de agua caliente, se deduce que la temperatura del agua que entra al acumulador depende de la que proviene del campo de colectores térmicos $T_{\text {colectores }}$. Es, así pues, que nuestra tercera función objetivo es:

$$
\min \left(t_{T_{\text {colectores }} \rightarrow T_{\text {colectores, nominal }}}\right) \rightarrow 0
$$

De esta manera, se concluye que se debe diseñar un controlador para el circuito primario de agua caliente que permita cumplir lo dicho en la expresión (7).

\subsection{Estrategias de Control escogidas}

De acuerdo con el alcance de TRNSYS, se cuenta con tres estrategias de control para ser empleadas al instante [8],[9]. Estas son:

- El control diferencial (On-Off).

- FeedBack Iterative Control (FB).

- El control proporcional-integral-derivativo (PID).

De acuerdo con los estudios revisados (§1), la estrategia que resalta a utilizar, de las antes mencionadas es control PID, sin embargo, el conocimiento de la función de transferencia del proceso que se desea controlar es indispensable, la cual está fuera del alcance de este trabajo.

Por otro lado, en la estrategia de control FeedBack Iterative Control (FB) que no es más que un control flotante en lazo cerrado, también se hace necesario, pero no indispensable contar con dicha función de transferencia para obtener los cálculos de estabilidad del proceso.

La mejor forma de obtener la función de transferencia es con los datos experimentales obtenidos de una medición en tiempo real. Dicho de otra forma, como se está trabajando con sistemas térmicos transitorios, tomando en cuenta las variables de entrada y salida de la función de transferencia, deseada los datos obtenidos experimentalmente, serían los más adecuados para la obtención de dicha función.

Debido a que el sistema de absorción no ha sido implementado todavía y en las estrategias de control, excluyendo el control PID, no se necesita indispensablemente la función de transferencia del proceso, se decidió escoger como estrategias a utilizar, el control Diferencial On-Off y el Control flotante (FeedBack iterative control). Salvo que, para la segunda estrategia, se utilizará el proceso de ensayo y error para cumplir con el objetivo.

\subsection{Diseño del sistema de control para el circuito de agua caliente}

Debe notarse que los controladores implementados para el sistema de control vienen incorporados en TRNSYS. El diseño del sistema de control se basa en la modificación de los valores por defecto, tomando en cuenta lo mencionado en \$2.2.

Los valores finales propuestos para los parámetros de los controladores se obtuvieron a partir de iteración manual tomando como objetivo primordial que la capacidad del Chiller de absorción lograra suplir la carga térmica de las zonas acondicionadas en todo momento.

\subsubsection{Circuito primario}

Partiendo de lo dicho en la sección anterior, el sistema de control en el circuito primario (ver figura 3) está destinado a reducir el uso del calentador auxiliar por medio de la temperatura de los colectores. El control se decidió realizar sobre los dispositivos manipulables de este circuito: el encendido y apagado de la bomba de agua, manteniendo su flujo constante, y la adición de una válvula aliviadora. Teniendo un controlador para cada dispositivo mencionado, la estrategia utilizada fue control diferencial On-Off (ver figura 4). En la figura 4, se indican las temperaturas utilizadas como entradas a los controladores y los dispositivos que son controlados.

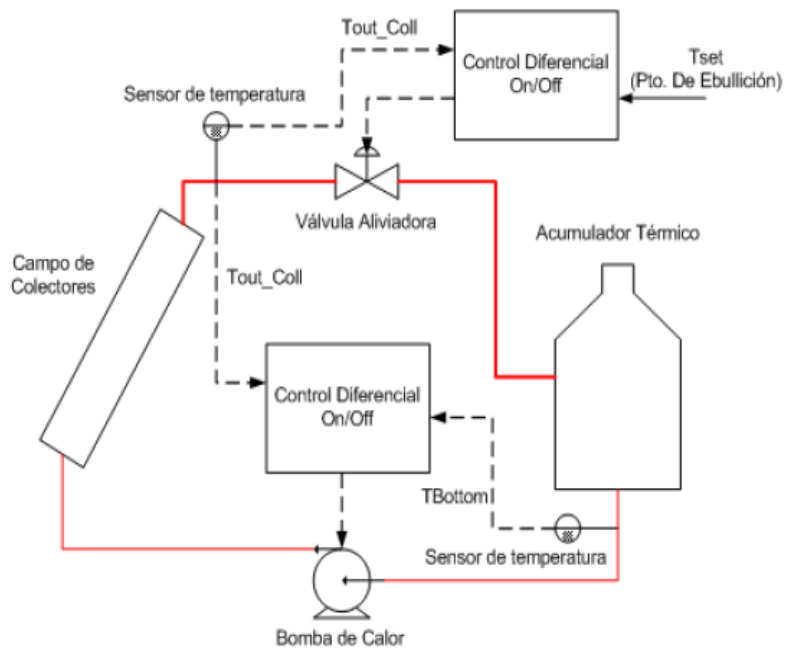

Figura 3. Circuito primario del agua caliente. Líneas rojas indican el paso por donde circula el agua caliente.

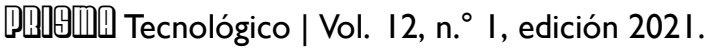




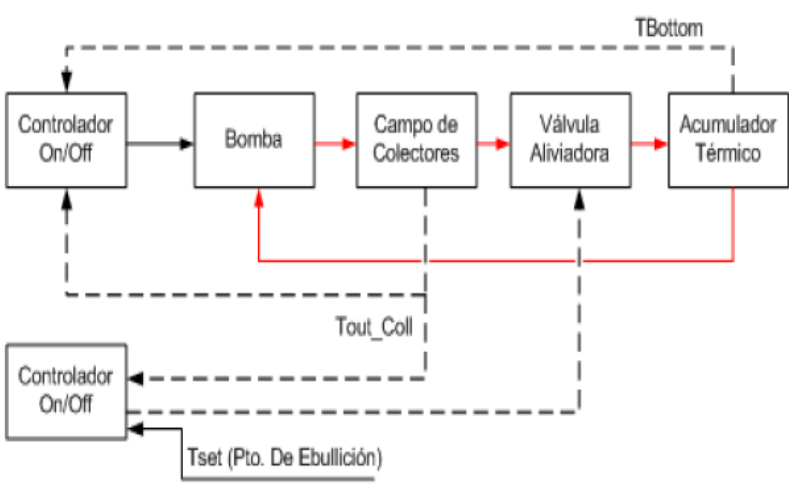

Figura 4. Esquema de la estrategia de control para el circuito primario del agua caliente. Líneas rojas indican el paso por donde circula el agua caliente (de la figura 3).

\subsubsection{Circuito secundario}

El sistema de control para el circuito secundario (ver figura 5) está destinado a mantener a la temperatura del agua que entra al generador en su valor nominal.

El control se decidió realizar sobre la válvula divergente (conocida como tempering valve) para la manipulación del flujo de agua que sale del generador, junto con la adición de una bomba de caudal variable.

También se coloca un controlador para el calentador auxiliar. Así, teniendo un controlador para cada dispositivo mencionado, las estrategias de control escogidas fueron: el control Feedback y el diferencial On-Off como se muestra en la figura 5 y 6 . En esta última figura, se indican también las temperaturas utilizadas como entradas a los controladores y los dispositivos que son controlados.

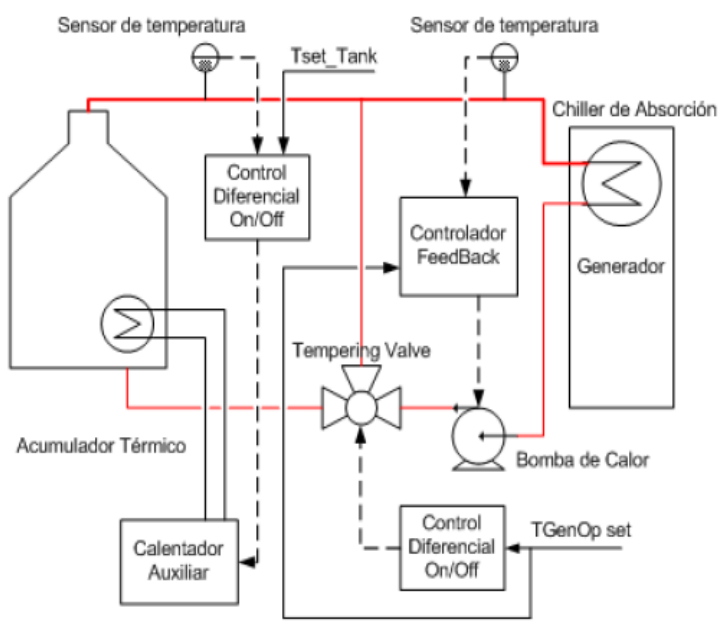

Figura 5. Circuito secundario del agua caliente. Líneas rojas indican el paso por donde circula el agua caliente.

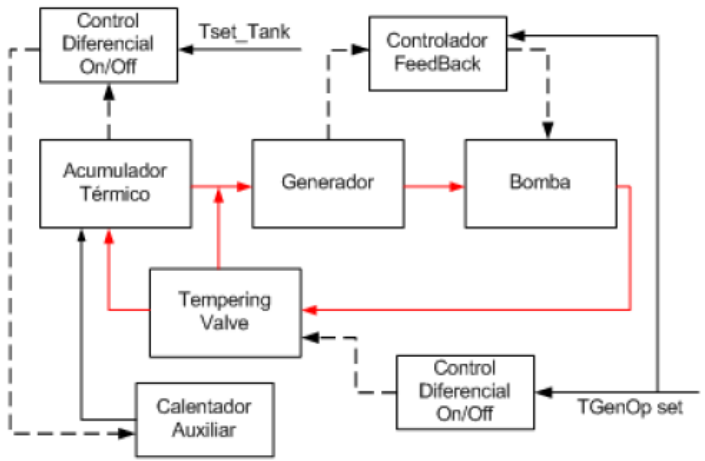

Figura 6. Esquema de la estrategia de control para el circuito secundario del agua caliente. Líneas rojas indican el paso por donde circula el agua caliente (de la figura 5).

\section{Resultados y análisis}

Comparando los resultados obtenidos presentados en las figuras 7 , con los obtenidos en la figura 9 se observa claramente que la estabilidad de las temperaturas de mayor importancia dentro del Chiller de absorción es alcanzada; los picos en $T_{E}$ (en color celeste) han sido completamente eliminados y $T_{G}$ (en color rojo) se encuentra dentro de los rangos nominales de operación, como se deseaba.

En las figuras 8 y 10, se observa que el consumo eléctrico aumentó considerablemente, debido al aumento en el uso del calentador auxiliar del acumulador térmico. Lo anterior es consecuencia de que uno de los objetivos propuestos, es el de minimizar el tiempo en el que la temperatura del agua que entra al generador llega a su valor nominal de operación deseado. Este tiempo pasa de ser, de dos días aproximadamente a 6 horas. Paralelamente este aumento del uso del calentador auxiliar se ve afectado por el valor de temperatura de operación del generador, el cual es de $80^{\circ} \mathrm{C}$ (antes de aplicar el sistema de control) sin embargo, se encuentra un valor mínimo óptimo para la operación segura del sistema de $85^{\circ} \mathrm{C}$ (después de aplicar el sistema de control).

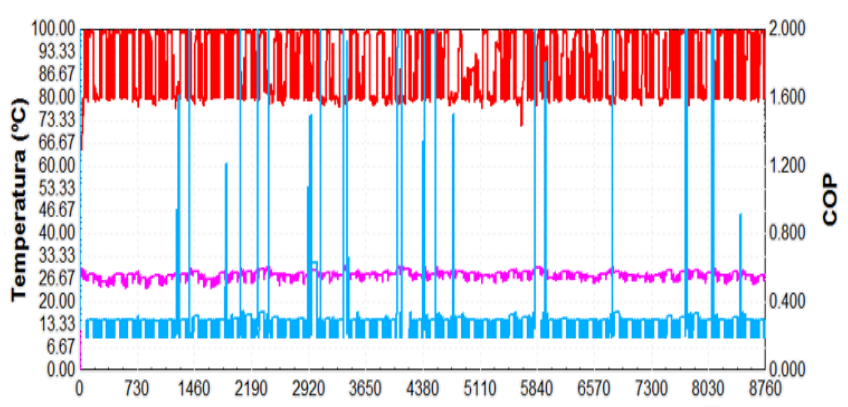

Figura 7. Resultado de simulación para un año, antes de aplicarle el sistema de control diseñado [3]. $T_{G}$ en rojo, $T_{T}$ en rosado y $T_{E}$ en celeste. 


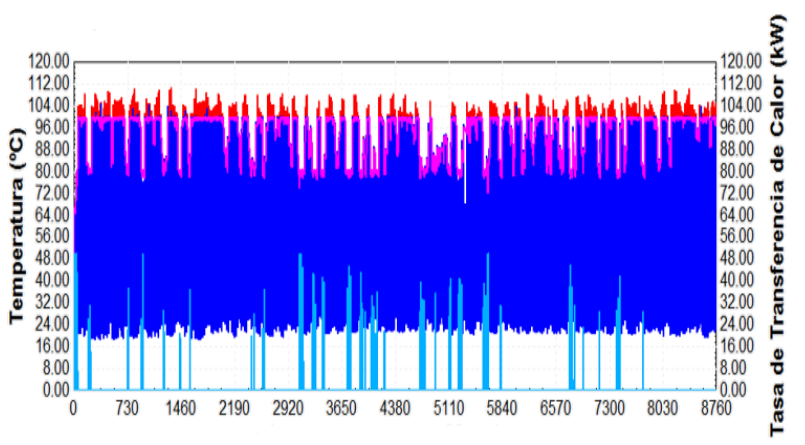

Figura 8. Resultado de simulación para un año, antes de aplicarle el sistema de control diseñado [3]. $T_{\text {colectores }}$ en rojo, $T_{\text {acumulador }}$ de salida en rosado, $\dot{Q}_{\text {aux }}$ en celeste y $T_{\text {val }}$ de salida en azul.

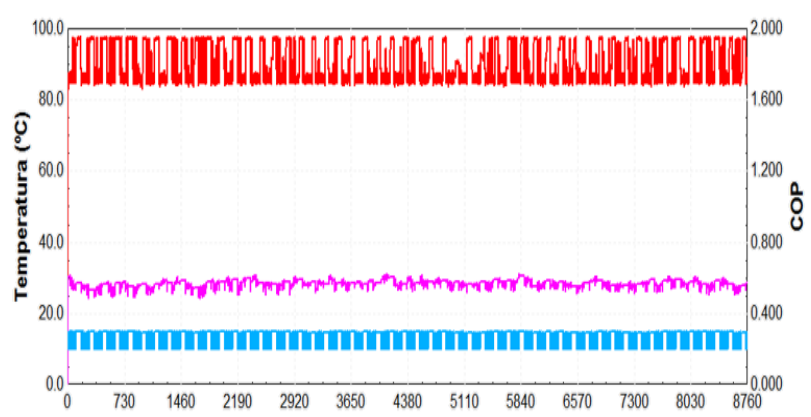

Figura 9. Resultado de simulación para un año, después de aplicarle el sistema de control diseñado. $T_{G}$ en rojo, $T_{T}$ en rosado y $T_{E}$ en celeste.

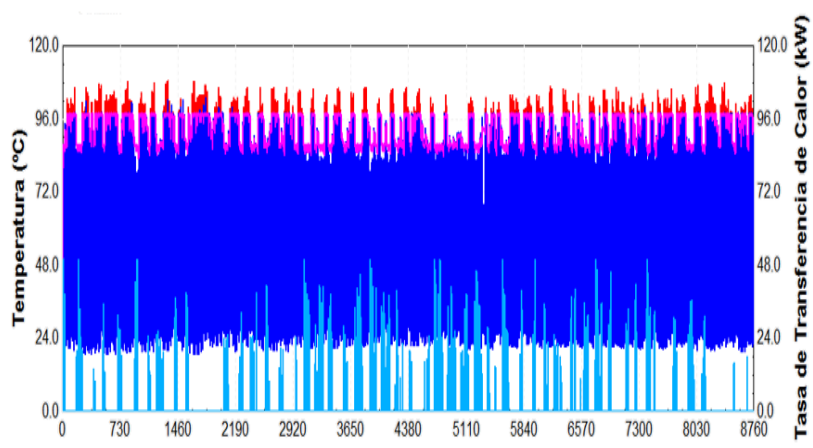

Figura 10. Resultado de simulación para un año, después de aplicarle el sistema de control diseñado. $T_{\text {colectores }}$ en rojo, $T_{\text {acumulador }}$ de salida en rosado, $\dot{Q}_{a u x}$ en celeste y $T_{\text {val }}$ de salida en azul.

En detalle, para $80^{\circ} \mathrm{C}$ se tiene un consumo de $8892,06 \mathrm{kWh}$ e interpolando para $85^{\circ} \mathrm{C}$ se tiene un consumo de $14683 \mathrm{kWh}$. Por el contrario, los resultados de este trabajo muestran que para $85^{\circ} \mathrm{C}$ se tiene un consumo de $22015,52 \mathrm{kWh}$ (sólo para el calentador auxiliar), el cual representa un aumento del $33,3 \%$ en el consumo eléctrico de la instalación.

Las señales de control de los controladores del circuito secundario de agua caliente son presentadas en la figura 11, para el 1ero de enero. Aquí, se muestra la operación del Chiller (en celeste) y la del calentador auxiliar (en rosado). Podemos observar que el calentador auxiliar se mantiene encendido durante todo el tiempo de operación del Chiller.

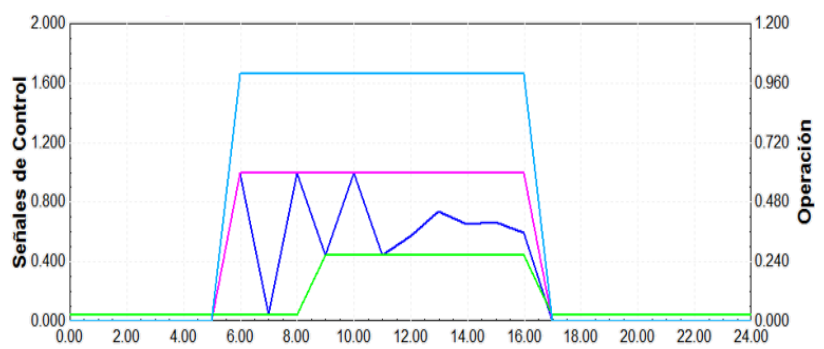

Figura 11. Señales de control, resultado de simulación para el 1ero de enero. Operación diaria del Chiller en celeste, operación del calentador auxiliar en rosado, señal de control de la bomba del sistema secundario en azul y señal de control para el controlador Feedback.

En las figuras (12 y 13), se presentan con la finalidad de comparar el tiempo de respuesta de la temperatura del agua que entra al generador (en rojo). Este tiempo es el que le toma a esta temperatura llegar a su valor nominal, desde el arranque del sistema. En la figura 12, se aprecia que la temperatura del agua que entra al generador no logra alcanzar valores nominales $\left(T_{G}\right.$ $>80^{\circ} \mathrm{C}$ ) luego de 2 días de haber arrancado el sistema. En cambio, luego de aplicar el sistema de control (figura 13), se aprecia que la temperatura del agua que entra al generador alcanza valores nominales luego de 10 horas de haber sido arrancado el sistema.

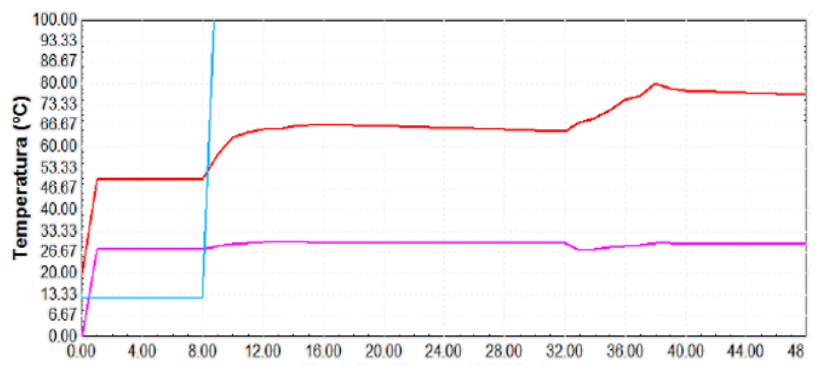

Figura 12. Resultado de simulación para el 1ero de enero, antes de aplicarle el sistema de control diseñado [3]. $T_{G}$ en rojo, $T_{T}$ en rosado y $T_{E}$ en celeste.

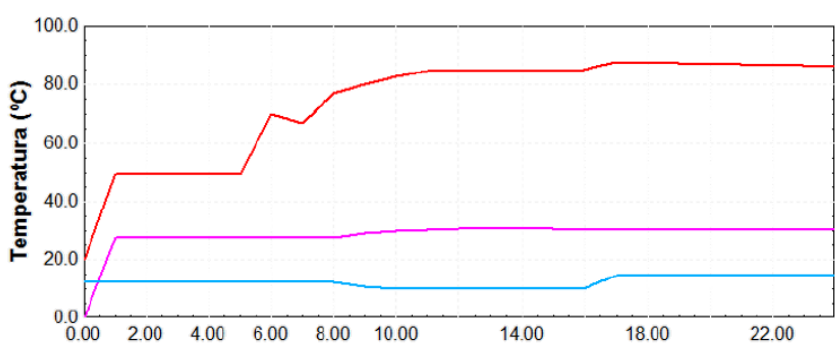

Figura 13. Resultado de simulación para el 1ero de enero, luego de aplicarle el sistema de control diseñado. $T_{G}$ en rojo, $T_{T}$ en rosado y $T_{E}$ en celeste.

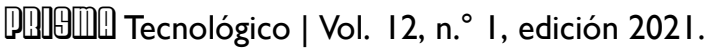




\section{Conclusión}

En la búsqueda de nuevas alternativas que ayuden a la reducción del consumo eléctrico destinado al proceso de acondicionamiento de aire en la República de Panamá, un estudio fue realizado en la Universidad Tecnológica de Panamá por el centro de investigación CINEMI. Dentro de este estudio, se propuso el diseño de una instalación solar acondicionadora de aire por absorción utilizando la herramienta de simulación TRNSYS, para luego ser implementado. El resultado de las simulaciones del diseño propuesto, para utilización bajo las condiciones climáticas de Panamá, predice que en ciertos momentos del año el Chiller de absorción presentará comportamientos fuera de su rango nominal (figura 7). Esto último, involucraría fallos en el Chiller ya que, al no operar dentro del rango nominal, se puede presentar el mayor problema que tienen estos dispositivos: la cristalización del $\mathrm{LiBr}$, lo cual ocasiona obstrucciones en las tuberías. Aquí, se propuso el diseño de un sistema de control de simple implementación, utilizando TRNSYS; el cual se basó en el análisis de reducción de parámetros a controlar y a optimizar. Este sistema de control ha contribuido satisfactoriamente a que el Chiller de absorción trabaje dentro del rango nominal durante todo el año (figura 9).

Lo anterior, condujo a un aumento en la utilización del calentador auxiliar de la instalación, aumentando su consumo eléctrico global en un $33,3 \%$.

Panamá al ser un país ubicado cerca de la línea ecuatorial, presenta una cobertura solar junto con una radiación solar aprovechable, potencialmente alta. Así, el uso de sistemas acondicionadores de aire por absorción con asistencia solar, presentan un menor consumo eléctrico (en comparación con los sistemas convencionales, por compresión y absorción).

Una ventaja de los sistemas de absorción con respecto a los sistemas por compresión es que los de absorción desechan el calor al ambiente, a una temperatura más baja; lo cual reduce su impacto al medio ambiente circundante. Por el contrario, los sistemas de absorción alcanzan la temperatura deseada en menor tiempo con un coeficiente COP mayor, que los segundos (alrededor de 0,8).

El realizar las simulaciones con la herramienta TRNSYS, llevó a encontrar limitaciones en cuanto a las estrategias que se implementaron en el diseño del sistema de control. Por ejemplo, no se pudo utilizar la estrategia PID ya que, se necesita la función de transferencia del proceso que se desea controlar. Esta función de transferencia se obtiene de forma experimental, para lograr resultados más exactos en las simulaciones. Sin embargo, la instalación no ha sido implementada todavía.

\section{Recomendaciones}

Los picos que se presentan en el resultado de la potencia del calentador auxiliar (en celeste, figura 10), aparecen con mayor frecuencia en los arranques del Chiller (cuando comienza un día de trabajo). Una alternativa para eliminar estos picos y así reducir el consumo eléctrico, sería un sistema "híbrido", el cual trabaje con fuente auxiliar eléctrica y de gas. La fuente auxiliar de gas se utilizaría para los arranques y la eléctrica en el resto de la operación. Por ejemplo, en una simulación realizada con un sistema que tenga una fuente auxiliar solamente a gas, dio como resultado una reducción del $28,74 \%$ en el consumo eléctrico anual.

Otra alternativa incluye el estudio sobre un sistema híbrido, que contenga no sólo ambos tipos de fuente auxiliar, sino que conste de un sistema de compresión mecánica de vapor refrigerante y uno de absorción.

Por último, una vez el sistema haya sido implementado, se propone diseñar el sistema de control haciendo uso de las funciones de transferencia de los diferentes dispositivos dentro del sistema solar acondicionador de aire por absorción.

\section{Referencias}

[1] (2008) Serrano, W.J. Panamá derrocha energía en aires acondicionados y refrigeración [en línea], http://burica.wordpress.com/2008/10/10/panamaderrocha-energia-en-aires-acondicionados-y-refrigeracion/.

[2] Programa de Normalización para la Eficiencia Energética. p. 9.

[3] E. Quintana, "Diseño y Simulación de un Sistema de Acondicionamiento de Aire por Absorción utilizando como Medio Energético Principal o Parcial Colectores Solares Térmicos", I+D Tecnológico, vol.9, n² 2, pp. 48-58, 2012.

[4] E.F. Camacho, et al., "A survey on control schemes for distributed solar collector fields. Part I: Modeling and basic control approaches". Solar Energy, 81(10), pp. 1240-1251, 2007.

[5] A Núñez-Reyes y C. Bordons, "Predictive control of a solar air conditioning plant with simultaneous identification", Proceedings of the 44th IEEE Conference on Decision and Control, and the European Control Conference, pp. 1355-1360, 2005.

[6] L.A. Bujedo, J. Rodríguez, and P.J. Martínez, "Experimental results of different control strategies in a solar air-conditioning system at part load". Solar Energy, 85(7), pp. 1302-1315, 2011.

[7] X.Q. Zhai, et al., "A review for research and new design options of solar absorption cooling systems". Renewable and Sustainable Energy Reviews, 15(9), pp. 4416-4423, 2011.

[8] TRNSYS 17 Manual - Mathematical Reference. Vol. 4. 2010.

[9] (2013) Wisconsin, U.o. TRNSYS 17 [en línea], http:/1/sel.me.wisc.edu/trnsys/features/features.html. 